NISTIR 7940

\title{
Dexterous Manipulation for Manufacturing Applications Workshop
}

Joe Falco

Jeremy Marvel

Elena Messina 


\title{
Dexterous Manipulation for Manufacturing Applications Workshop
}

\author{
Joe Falco \\ Jeremy Marvel \\ Elena Messina \\ Intelligent Systems Division \\ Engineering Laboratory
}

http://dx.doi.org/10.6028/NIST.IR.7940

June 2013

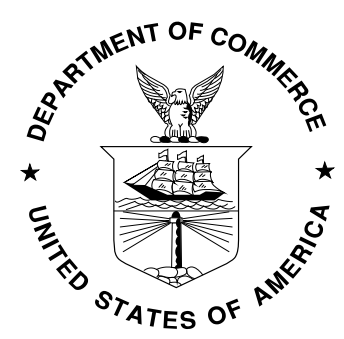

U.S. Department of Commerce

Cameron F. Kerry, Acting Secretary

National Institute of Standards and Technology Patrick D. Gallagher, Under Secretary of Commerce for Standards and Technology and Director 


\title{
Dexterous Manipulation for Manufacturing Applications Workshop
}

\author{
Workshop Organizers \\ Joe Falco, Jeremy Marvel, Elena Messina \\ National Institute of Standards and Technology
}

\section{Panel Experts}

Technical Session 1: Industry Perspective on Dexterous Manipulation

Mark Lewandowski, Procter \& Gamble (P\&G)

Craig Battles, Boeing

Clay Flannigan, Southwest Research Institute (SwRI)

Technical Session 2: Robotic Hands

Jesse Hayes, Schunk

Jean-Philippe "JP" Jobin (for Samuel Bouchard), Robotiq

Jeremy Fishel, SynTouch

Technical Session 3: Dexterous Arms

Michael Gerstenberger, KUKA Robotics

Alex Goodwin, Rethink Robotics

Tim DeRosett (for Erik Nieves), Yaskawa Motoman Robotics

Workshop Date:

January 24, 2013 


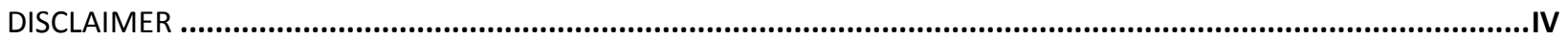

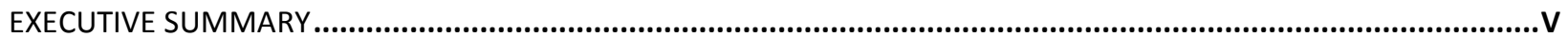

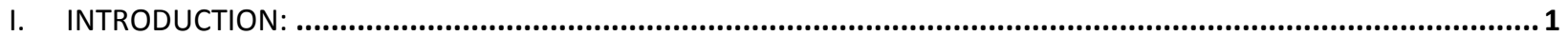

II. TECHNICAL SESSION 1: INDUSTRY PERSPECTIVE ON DEXTEROUS MANIPULATION .......................................

A. Robotic Gripping and Manipulation Needs at P\&G - Mark LeWandowski, Procter and Gamble (P\&G) ................2

B. AsSembly ANd Automation TeChnology - Craig BATTLES, Boeing ...............................................................

C. Material handling in Unstructured EnVIRonments: Challenges and Opportunities for Small and Medium

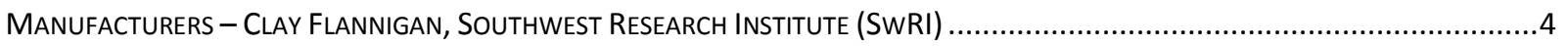

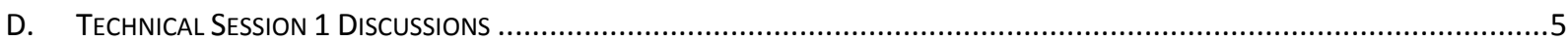

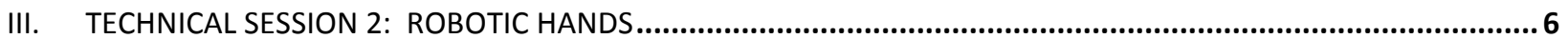

A. Robotic Gripping: What IS The FutURe? - JeSSE HAYES, SCHUNK ...............................................................

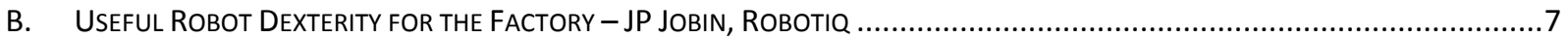

C. Multimodal Tactile Sensing And Industrial Applications - Jeremy Fishel, SYNTOUCh .......................................

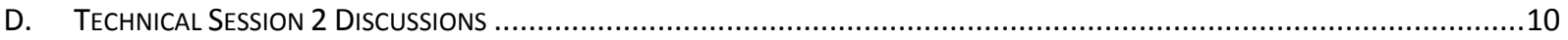

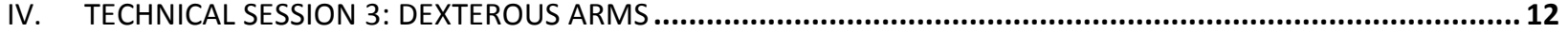

A. Force Guided Assembly With the KUKA LightWeight Robot - Michael Gerstenberger, KUKA Robotics................12

B. Part Manipulation using Sensing and Force Control - Alex GoodWin, Rethink Robotics................................12

C. Bilateral Manipulation Applications In Industry - TIM DEROSETt, YASKAWA Motoman RoBotics ........................13

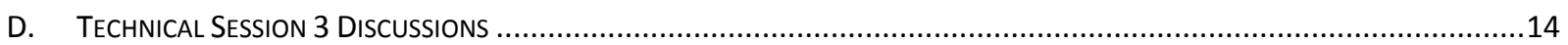

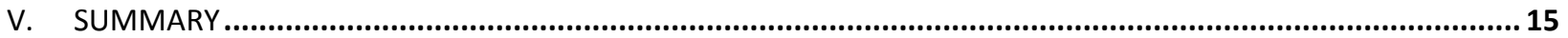

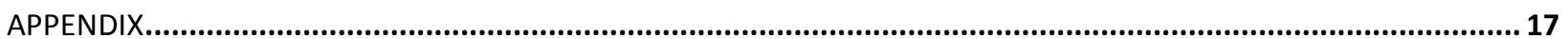

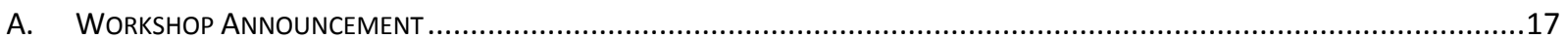

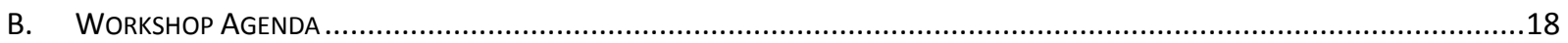




\section{Disclaimer}

Certain trade names and company products are mentioned in the text or identified in certain illustrations. In no case does such an identification imply recommendation or endorsement by the NIST, nor does it imply that the products are necessarily the best available for the purpose.

The opinions expressed in this Workshop Report are those of the workshop participants and are not the official opinions of NIST. The summaries of the presentations have been reviewed by the speakers and the summaries reflect the speaker's main points. 


\section{Executive Summary}

Manufacturers face many challenges in implementing next-generation automation, especially when their production mix involves low volumes of parts with high mixtures of part types. Small batches, which might include even batches of one, require quick turnover rates and the ability to reprogram a multiplicity of involved processes. This challenging environment is aggravated for small and medium sized manufacturers who already have limited capital and high labor costs. Coupled with the low volume and high mix processes, this makes installing, supporting, and modifying dedicated automation systems prohibitively expensive and structurally impractical. Automation requirements to support these manufacturing needs are beyond the capabilities of even the most sophisticated robots, and present major obstacles to moving robots into small and medium-size enterprises. One of the essential capabilities needed to address the challenges in next-generation automation is believed to be robots that are equipped with dexterous manipulators. ${ }^{1}$ Dexterous manipulation has been defined as "an area of robotics in which multiple manipulators, or fingers, cooperate to grasp and manipulate objects."2

The National Institute of Standards and Technology (NIST) organized an industry workshop in conjunction with the 2013 Automate and ProMat trade shows in Chicago, IL. The goals of the workshop were to address the application of dexterous robot technologies to meet the application needs of small batch production, and to promote the greater theme of applying dexterous robotics toward flexible manufacturing. The workshop consisted of three technical sessions featuring presentations from industry leaders in dexterous grasping, robot arm technology, and flexible manufacturing. Each session concluded with an open-format panel discussion where all participants discussed the needs and opportunities for dexterous manipulation in next-generation manufacturing systems.

Challenges faced by small and medium enterprises were articulated, as were those of large-scale manufacturers. Although there were some differences in emphasis, overall some universal needs emerged. These include the need for more easily instructing robots to perform manufacturing operations tasks in challenging and dynamic environments and the desire for more general-purpose end-of-arm tooling that is able to grasp and manipulate a broader range of parts with high reliability. A consumer goods manufacturing perspective characterized next generation requirements as being able to achieve the dexterity to grasp many objects with different shapes in any number of orientations quickly, adeptly, and decisively. These automation systems must incorporate force and tactile sensing enabling a flexibility that is "blind to shape" in order to use the sense of touch to acquire as humans do. Robotic systems with "human-like" capabilities and high reliability are required. "Human-like" refers to attaining comparable functionality for grasping and manipulating a large array of products, not necessarily having an anthropomorphic structure or design. Aircraft manufacturing key challenges were described as the need for portable automation to support the large footprint of an aircraft assembly and confined space access at tolerances on the order of $0.25 \mathrm{~mm}$. Some of the biggest drivers of robot dexterity were described as safety, quality control, cost reduction, and throughput. Safety was reported by one presenter as a major driving factor in recurring costs due to ergonomic factors resulting in repetitive strain, over-extension, and over-exertion.

Intelligent gripping technologies include sensing for force control, adaptable gripper pads, and multifunction grippers for handling multiple parts within a defined process. While it is apparent that conventional gripping is transitioning from "single product" gripper to "single system" robotic hand

1 "A Roadmap for U.S. Robotics: From Internet to Robotics" , 2013, http://roboticsvo.us/sites/default/files/2013\%20Robotics\%20Roadmap-rs.pdf

2 Okamura, A.M.; Smaby, N.; Cutkosky, M.R., "An overview of dexterous manipulation," Robotics and Automation, 2000. Proceedings. ICRA '00. IEEE International Conference on , vol.1, no., pp.255,262 vol.1, 2000. doi: 10.1109/ROBOT.2000.844067 
technologies, some believe that reactive grasping capabilities will someday allow for blind grasping of an object without the use of a vision system. Others believe that the future of robotic grasping is tighter integration of perception data, grasp planning algorithms, sensing systems, and gripper mechanics. There are countless robot hand prototypes being developed at universities thanks to the advent of rapid prototyping processes A few commercially available robotic hands are starting to make their way into manufacturing environments. In addition, tactile sensing technologies are progressing beyond just being able to sense forces normal to, and at the resolution of a tactile sensor pad. New tactile sensing technologies are in development with capabilities to resolve the directions of forces as well as sense shear forces. In addition, other sensing capabilities such as temperature and vibration are evolving that are expected to make tactile sensing equivalent to or even better than that of the human hand.

Advances in robot arm technology are also being made to support the dexterity needed for flexible manufacturing by integrating force sensing technology directly into robot joints, as opposed to the use of force transducers attached to a tool flange. Using force control effectively eases application restrictions on part uncertainty, allows for lower accuracy in part placement and fixturing, and supports force-guided search strategies that enable assembly operations such as insertions, gear meshing, and snap fitting. In addition, these new robot arm technologies are being designed with force control and compliance characteristics that enable them to work alongside people performing human-scale tasks at human cadence, eliminating the need for safety cages. Dual 7 degree-of-freedom arms with coordinated motion and capabilities maximize volumetric reach and orientation flexibility.

Discussions considered the need for performance metrics and test methods for this new generation of robotic hands and arms, as well as for integrated systems consisting of both arms and hands. A framework to evaluate tasks and parts was identified as a means to characterize the effectiveness of solutions and the use of competitions was discussed as a viable mechanism to drive research and development. A rudimentary set of performance metrics and associated test artifacts were identified as useful tools in helping to progress such a young sub-field of robotics. Workshop participants agreed that dexterous robotics will continue to be a topic of active research due to the sheer volume of work coming from universities with the unanswered question being: how will industry drive the transition of research solutions into real world solutions?

The results of this workshop will be used as input for a roadmap being drafted by NIST aimed at progressing standards and measurement science in the area of dexterous manipulation. This report as well as the forthcoming roadmap will be made available on the Dexterous Manipulation for Part Grasping and Assembly project website (http://www.nist.gov/el/isd/ps/dexmanpartgraspassem.cfm). 


\section{Introduction:}

On January 24, 2013, NIST hosted a technical workshop during the joint Automate and ProMat trade shows at the McCormick Place convention center in Chicago, IL. The goals of the workshop were to address the application of dexterous robot technologies to meet the application needs of small- and medium-sized manufacturers, and to promote the greater theme of applying dexterous robotics toward flexible manufacturing.

The Dexterous Manipulation for Manufacturing Applications Workshop featured presentations from consumers of dexterous robotics, next-generation dexterous robot manufacturers, and dexterous robot hand technology leaders. Each of the three technical sessions featured three speakers who collectively represent the cutting edge in dexterous robotics use and development, each presenting different perspectives on the session topic. Following each set of presentations, the invited speakers were asked to return to the stage for an expert panel discussion of the impacts, challenges, and necessary next steps of their respective technological needs and solutions.

This report summarizes the presentations shared at the Dexterous Manipulation for Manufacturing Applications Workshop, and captures the discussions held during the expert panel sessions. Section II provides synopses of Technical Session 1, which focused on the industry users' perspective on dexterous manipulation. Section III discusses Technical Session 2 on the state of the art of dexterous robot hands. Section IV discusses Technical Session 3 on the perspectives of dexterous robot arm manufacturers. 


\section{Technical Session 1: Industry Perspective on Dexterous Manipulation}

\section{A. Robotic Gripping and Manipulation Needs at P\&G - Mark Lewandowski, Procter and Gamble (P\&G)}

$P \& G$ is the largest consumer product manufacturer in the world with over 2000 products. The presentation highlighted manufacturing processes at $P \& G$ that are in need of robotic grippers and manipulators that are human-like, reliable, and affordable. Mark described an existing manufacturing process of manually placing bottles on a conveyor system for subsequent automated dispensing of product followed by additional manual operations for capping the bottles. This single dispensing line handles 50 to 100 product batches, each consisting of different part variations in terms of shape and size. The product variations are not dictated by automation constraints, but are instead artistically driven by the consumer goods marketing community, resulting in bottle shapes that are round, square, and even helical-shaped, and often include irregular surfaces. The diversity of bottle shapes and sizes makes automation using currently available technologies infeasible.

A video of a packing line was shown in which workers performing manual operations receive parts (bottles or caps) in boxes that typically incorporate interior cardboard dividers. These cellular packaging schemes do not fully constrain parts, which results in shifted, tipped, rotated or even missing components due to handing prior to arrival at the production line. As a result, readily available robot technologies are unable to supplement the efforts of existing assembly workers.

Gripper and manipulation needs at P\&G include "human-like” capabilities in terms of functionality, but this does not necessarily mean they must be anthropomorphic in nature. Such systems must have the dexterity to acquire objects in any number of orientations with the speed and actuation necessary to grasp adeptly and decisively. The systems must incorporate force and tactile sensing to make grasping adjustments on the fly and to mimic the human's capability to feel their way into a box with a flexibility that is "blind to shape." Mark believes that grippers need to be greater than $99 \%$ reliable so they are not the weakest link in the processing chain. Grippers must hold parts securely during high-speed moves such that the mean time between failures is on the order of every 200 to 300 minutes, a measure Mark stressed as being on the order of hours, not seconds or even minutes. Mark suggested that the cost of these robotic solutions should be competitive with low-wage labor. Universal-type gripper costs can be justified if they yield flexibility and reliability when compared to the high costs of custom gripping solutions designed for individual parts. He described the need for grippers that fall between custom grippers, which are highly effective for a single design, and universal grippers, which can grip a large array of products. Ideally, the gripper would be transferable between limited product lines and still provide useful grasping capabilities.

Mark discussed the need to assess the actual cost of existing automation solutions as compared to manual labor. In an example situation, integration and support costs continually add to the price of grip solutions, and the automation of these processes is losing ground to the lower labor rates found in other countries where production lines typically run 14/7 (fourteen hours per day, seven days a week) or 24/5 with multiple shifts. Mark concluded by stating that breakthroughs in capability and cost are needed to make low-speed manual operations "automation affordable.” 


\section{B. Assembly and Automation Technology - Craig Battles, Boeing}

At the beginning of Craig's presentation, a variety of two-part aircraft fasteners were circulated among the audience. These fasteners are typical of those used in constructing Boeing aircraft, and were intended to help the audience realize the complexity of the parts used in the aerospace industry. In addition to part complexity, Craig explained that the process of inserting, threading, and tightening these fasteners into an airplane is difficult, as is the process of drilling holes with required tolerances on the order of $\pm 0.25 \mathrm{~mm}$ ( $\pm 0.010 \mathrm{in})$ in size and location.

Craig identified the biggest drivers of robot dexterity and automation needs at Boeing as safety, quality control, cost reduction, and throughput. Typical assembly tasks include fastening, loading/unloading, and sealing. Each has different challenges for quality control and different associated costs. The safety risks to humans include injury due to repetitive tasks, the use of tools, and exposure to hazards within the automation environment. Drilling, fastening, and assembly are the principal sources of manufacturing defects. While small numbers and types of defects are tolerable, the accumulation of too many defects leads to the scrapping of large components and aircraft sections.

Craig explained that Boeing's costs are categorized as being recurring or non-recurring. Recurring costs account for $75 \%$ of Boeing's costs, and are often passed along to the customers. Such costs include day-to-day operational costs such as materials, direct factory labor, support labor, and reworking parts or products. Non-recurring costs are incurred at specific milestones for a particular product line. These costs include research and development, design/engineering, tooling design, and capital, and account for approximately $25 \%$ of Boeing's costs. From a cost perspective, safety is a major driving factor in recurring costs. Two-thirds of all workers' compensation cases are ergonomically based, and are typically caused by instances of repetitive strain, over-extension, and over-exertion. Tool design and fixturing consumes the largest percentage of total investment over the lifetime of a product. The fixturing for the assembly of wing structures is 4 stories tall and can handle 60 meter wing spans. He went on to describe a flexible fixturing system developed at Boeing consisting of small manipulators that are used to latch onto a large part at specific positions and are controlled simultaneously to manipulate the fixtured part.

Craig explained that the current state of the art of aircraft construction poses unique challenges for robotics and automation. Because of the large footprint of an aircraft assembly, there is a need for portable automation solutions where systems can perform precision drilling and fastening of large areas and volumes. There are also requirements for confined space access where dexterous robots would be inserted through small holes. These small holes are currently accessible by workers, and are described as a close fit for an average sized human. In addition, robotic cleaning, sealing, painting, and surface preparation require precision control and sensor feedback. There is also the need for flexible fixture designs to accommodate shifts in product lines. At the scale of manufacturing aircraft, a dexterous "hand" could be comprised of a series of coordinated robots each acting as a finger.

Boeing's vision for the next generation of robotics includes technologies to support localized position and sensing, synchronized and coordinated robot platforms, swarm robotics, and adaptive control. Craig mentioned that common end effector interfaces are needed for robotic tooling, automated cleaning, and sealing and painting systems. There is also a need for single-sided fastening to support current fastening systems that require a manual worker on each side of the wall being fastened, improved crawler technology, reconfigurable mobile tooling, safe man-machine interfaces, in-process inspection, and automated error proofing. 


\section{Material Handling in Unstructured Environments: Challenges and Opportunities for Small and Medium Manufacturers - Clay Flannigan, Southwest Research Institute (SwRI)}

Clay began his presentation by making note of the steady increase in complexity of manufacturing tasks since the start of industrial robots in 1961 in terms of part clutter, overlap, and uncertainty. He explained the degrees of complexity associated with material handling using a method of characterizing this class of problems with four examples: structured material handling, semistructured material handling, bin picking, and unstructured material handling. He described structured material handling as a single rigid object fixtured for handling by a robot and picked up based on a predetermined part location. Semi-structured material handling consists of locating a single type of rigid object constrained on a specified object planar surface, which Clay defines as a 2 degree-of-freedom (DOF) pose. Multiple instances of the object types do not overlap, indicating a state of low clutter. A vision-based perception system provides uniform, controlled lighting, and the objects have diffuse reflectance and opaque transmittance. He described this classification as that of a $2 \mathrm{D}$ vision-guided material handling system, often found in today's manufacturing systems. He then characterized the bin-picking problem as involving a single type of rigid object randomly piled in a bin, requiring a perception system to resolve the pose of each object instance to 6 DOF in high clutter. He noted that there are few existing robotic solutions that incorporate bin picking, and that these implementations use vision-based perception systems that rely on uniform and controlled lighting, although the randomly oriented objects may have specular reflectance and opaque transmittance. The final example presented was that of an unstructured material-handling environment, in which many different object types (of both rigid and deformable structure) are randomly oriented, requiring resolving the pose of each object to $6 \mathrm{DOF}$ in a highly-cluttered environment. The environment is not well controlled in that it has variable lighting, and parts have both diffuse and specular reflectance, and both opaque and transparent transmittance. These manufacturing environments are impractical to automate with today's technology, and Clay categorized them as requiring manual material handling operations using humans.

Clay went on to describe the automation challenges of small manufacturers as being characterized by low volumes of parts with a high mixture of part types, including some one-off components, with high changeover rates and a multiplicity of involved processes. Small manufacturers have limited capital for equipment purchases and high labor costs. This, coupled with the low production volumes and high mix processes, makes process automation prohibitively expensive and structurally impractical. Adding specialty staff and dedicating existing staff to support automation is equally impractical. The automation must adapt to human environments, and be simple to implement and maintain by these same humans in order to be effective in a small manufacturing environment.

Clay continued by describing the state-of-the-art in several technology areas that represent progress towards supporting small manufacturers. He began with the recognition of multiple objects, describing work to develop systems that recognize tens of parts using shape and color cues. While this technology shows promise, results are not even close to human performance in this area. Next, he described research efforts and gripping technologies capable of handling multiple objects by means of adaptive gripping using approaches such as under-articulated mechanisms, vacuum based products, and electrostatic and jamming technologies. He characterized research results in grasp planning for robotic hands as being still too complex and time consuming, and explained that the problem of grasping is not independent of the perception component. He also stated that deformable and transparent objects pose their own challenges for part recognition and manipulation. 
Clay posed other considerations for active research and next-generation dexterous robot solutions, such as human interaction, safety, and ease of programming. He stated that ease of programming has been the biggest field of active robot research, and has seen the most changes in the past 50 years. In conclusion, Clay described a SwRI robotic research project in material handling using four types of rigid objects on display at the Automate trade show. This project produced a system capable of 3DOF pose estimation in a medium clutter environment with controlled lighting, diffuse reflectance, and opaque transmittance optical characteristics.

\section{Technical Session 1 Discussions}

At the beginning of the technical session, the question was posed: what metrics exist for evaluating technologies, and what are the methods for evaluation? In response, the panel concluded that current methods for evaluating technologies are very much empirical with significant trial and error, and are currently not treated with the rigor of hard science. Ideally the industry would come up with a framework to evaluate the processes and parts in order to characterize the effectiveness of potential solutions, as well as the conditions under which these tasks are being performed (e.g., lighting and part presentation). The framework introduced in Clay's presentation is one example of how this can be done. The panel then discussed some possible ways that the parts or environments could be classified. One participant suggested that a more comprehensive list would require stakeholder and industry input. A question was posed as to what metrics and languages currently are or could be used for describing the results of empirical tests to avoid constantly recreating the wheel. The panel concluded that processes and language for knowledge transfer simply do not exist. The discussion continued with the need for a common vocabulary for field metrics to characterize automation systems and environments.

A point was made that, in the case of gripping, the majority of academic research is focused on grasping solutions with 5-fingered hands, not on vacuum or other traditional gripper technologies where the characterization of technologies for different grasping tasks simply does not exist. More holistic approaches may actually be warranted to solve different classes of problem. Much of the testing performed today is art, not science. For instance, an integrator or vendor of a suction gripper system uses the part to be grasped to perform qualitative tests to determine which suction cup/vacuum system works best on a particular part. Metrics are needed for characterization and communication across large organizations but it is difficult to develop data models that capture salient characteristics of parts.

A question was posed with regard to the usefulness of challenges (e.g., the Defense Advanced Research Projects Agency, DARPA, challenges) to help solve problems in the area of dexterous manipulation aimed at industrial applications. One panelist indicated that holding challenges was possible, but not in the format typical of the DARPA competitions. He indicated that the difficulty of such a competition lies in the design. It would be difficult to create challenges that are agnostic to industries and manufacturers. The panel then agreed that developing a challenge would be difficult and that a given challenge must include both perception and manipulation; they cannot be separated.

The question was asked as to what means exist for making connections between the current needs of manufacturing and possible commercial solutions. Pathways do not currently exist for matching user needs with research interest. Ultimately, scale drives solutions. While this is a good field for research, the current manufacturing infrastructure is not in a good position to support it. Even internally within a company, simultaneous efforts may be focused on solving the same problem with 
the same variables, but there is no cross communication between units so the redundancy in tasks goes unnoticed.

Methods for transitioning solutions out of academia and into applied manufacturing are also lacking. Right now there is relatively little interest in research for hard industrial automation problems; instead researchers are addressing softer problems like assistive and medical robotics. Many similar problems are being addressed in personal care robotics and military robotics, but little is being done by the industrial sector. Other countries are now becoming more focused on industrial problems. The United States must follow suit. Technology transfer is also recognized as a big problem across sectors.

\section{Technical Session 2: Robotic Hands}

\section{A. Robotic Gripping: What is the Future? - Jesse Hayes, Schunk}

Jesse's presentation described the evolution of robotic gripping and introduced the next generation concepts of robotic hands. Jesse described the perfect gripper as one that is like the human hand: strong, sensitive, precise, flexible, reflexive, and intuitive to control. He indicated that the human hand is a great model to start with, but is sensitive and susceptible to damage. In industrial environments, damage and wear are common to industrial hardened gripping components.

Jesse described market trends for grippers in terms of pneumatic grippers that open and close and advances in technology for smarter gripping where sensors are added to achieve capabilities such as force control. He described the latter as intermediate technology leading toward the development of intelligent gripping for examination, flexibility, and adaptation. This technology heralds the beginning of robotic hands with the capability to adapt grasps to many different types of objects without the need for customized tooling.

The gripper industry is particularly adept at picking up the same part over and over using the part's own geometry to constrain it. However, as applications advance and customer requirements change, more flexibility and adaptability are needed. He described the problem of bin picking where a gripper must have the flexibility to handle the different exposed geometries for a single part type located in a bin. In some cases, the variations in exposed geometry are similar enough to allow using a conventional parallel gripper; however, bin picking in general needs adaptive gripping technologies.

Jesse then discussed industry needs in robotic grasping in terms of industry sectors where assembly automation requires high cycle times, robotic automation requires precision with tool change options, and material handling automation requires load capability, robustness, and damage prevention. He emphasized that all sectors require reliability. Flexibility is looked at as a future technology capability, while most of the focus on modern gripper design is placed on "before the hand" models (i.e., precursor technologies to anthropomorphic human-like hands). The gaps between current and future grippers are encompassed in mechatronics integrating electronic features with pneumatics, and solutions tend to seek improvements in energy efficiency.

He continued to describe the power density of pneumatic gripping as three times the cost, weight, and size to achieve the same performance using electric actuation, and stated that he believes that the industry is seeing a big drive to get away from pneumatics. Energy savings is another push away from pneumatics with short-term solutions of using point-of-use valves. There is a large loss in efficiency though air lines in comparison to the energy used at the gripper. To solve this problem in 
conventional pneumatic gripping solutions, micro valves are being fed locally to the gripper by a single air line, which reduces actuation time and air consumption. This typically results in a $100 \%$ to $200 \%$ increase in throughput and energy savings.

As a step towards adaptable gripping, Schunk has developed tools that have multiple functions. As an example, a tool was developed that can handle pallets, boxes, and slip sheets in a palletizing operation. As another example, material handling tooling including fingers with integrated circulating belts was developed to pick up and rotate boxes. Schunk expects there to be a lot of work in the area of multi-use gripping in the transition to robotic hand technology. Developments in sensor and finger technologies allow simple products to be applied to many applications. These technologies include analog-positioned and force-measuring fingers that are adjustable or compliant, and will ultimately reduce the need for tool changers. Jesse presented an example of a needle gripper designed to automatically adapt to different part geometries.

Schunk believes that the future of gripping will transition from "single product" gripper to "single system" robotic hand technologies. Reactive grasping or perception will tell the robot the size and shape of an object. Reactive grasping uses sensor information such as encoder data and tactile sensor data to blindly grasp an object without the use of a vision system. Jesse discussed the challenges of implementing robotic hands as reducing their weight to improve the robot's payload limitations, and modular so that interchangeable hands can handle different classes of part geometry. He described performance requirements of these hands in terms of position and force control as well as actuation speed. In addition, robot hands need to be energy efficient, adaptable, compact, commercially accepted, and safe. Schunk's most recent work in the area of robot hands includes the commercially available Schunk Dexterous Hand (SDH) and a new dexterous hand still in the prototype stages. The $\mathrm{SDH}$ is a 3 finger, $7 \mathrm{DOF}$ hand, with independently-actuated proximal and distal joints. Other features include modular fingers and joints as well as tactile sensing capabilities. The prototype robotic hand was developed by a company called Elumotion and is being industrially hardened and marketed by Schunk. An anthropomorphic design, the hand resembles the human hand in size, shape, and mobility, and has tactile sensing capabilities.

\section{B. Useful Robot Dexterity for the Factory - Jean-Philippe "JP" Jobin, Robotiq}

JP began by giving a brief history of his company. Robotiq was founded about five years ago with the idea of giving customers gripper products that can adapt to a variety of part geometries. Customers seeking automation capable of working with multiple part geometries, requiring manipulation by a single robot using multiple grippers and a tool change system, could investigate if a Robotiq adaptive gripper would be adequate for their needs and be more cost effective. In many cases, the cost of an adaptive gripper solution is lower than the cost of implementing a gripping system consisting of custom tooling for each part. JP explained that it is easy to justify the cost of robotic automation of a low mix of part types as compared to manual labor. However, as the mix of part types increases, the cost of automation grows exponentially, and quickly exceeds the cost of manual labor. This is why manufacturers need dexterity. These costs are directly associated with increased tooling, programming, floor and rack space, and changeover time. Many manufacturers have a high mix of parts, and require dexterity to make automated manufacturing work for them.

In the traditional approach to manufacturing, robots and grippers are basically movement generators with limited or no sensing capabilities. The use of vision has increased in recent years, and has improved many (but not all) applications. Force sensing is still very rarely seen in industry, and tactile sensing has never been seen in the hundreds of factories visited by Robotiq. Because minimal sensing is used, users need to rely on the mechanical repeatability of the system. This is a challenge 
when making flexible hands, because mechanical flexibility and mechanical repeatability do not go well together. To cope with the current limitations of robots, the production process is often changed to accommodate automation, and includes changing the features on the parts or the manner in which the parts are fed to the robot. Engineers break the system into digestible chunks, often missing integration relationships. For example, a more capable gripper can simplify the vision requirement, but this possibility may be missed because gripper choices are deferred to the very end of the automated system design cycle. Gripping should be considered at the beginning of the cycle and determined by a defined set of picking requirements such as stroke, grip force, speed, form factor, weight, material, repeatability, and sensing. Grasp stability requires that one take into account how well the part is held during robot motions; so, too, should the needs of manufacturing operations be considered.

Traditionally there is a trade-off between flexibility and performance. Conventional pneumatic grippers are strong, fast, repeatable, compact, reliable, and cost effective, but, in general, are not flexible. While the human hand has great capabilities and represents the best of both flexibility and performance, robotic hands that have been designed to be anthropomorphic lack speed, strength, and repeatability, which are three of the most important requirements in the manufacturing industry.

Robotiq's approach to robotic hand design focuses on a balance between flexibility and performance, resulting in gripping capabilities with decent performance and flexibility. New technologies in robot arms such as Rethink Robotics’ Baxter and Universal Robots’ arms seem to also be targeting flexibility with some performance tradeoffs.

What makes a good hand to enable dexterous manipulation in the factory involves finding the "sweet spot" in terms of flexibility, performance, and cost. Controlling a complex robotic hand is difficult, and requires good sensing capabilities that deliver data reliably, consistently, and in a robust and cost effective way. Considering that grippers pick thousands to millions of parts in a tough manufacturing environment, this poses a serious design challenge for tactile sensors that are the first line of contact. Algorithms must be developed to process sensor data and detect things such as part geometry or part slippage, which will open a world of applications just as vision has over recent years. Sensing will provide the necessary control sensitivity to reduce the burden on mechanical components, potentially lowering the cost of the mechanics needed in a robotic hand.

When trying to benchmark dexterity, the most meaningful unit to measure is the cost savings that it can provide. It would be useful to define dexterity in the context of manufacturing so that robot and component providers have a target to aim for. DARPA does this for the military, providing challenges that represent current problems that robotics should solve. NIST has started a project to benchmark dexterous industrial manipulators and hands. As an example of benchmarking dexterity, the Southampton Hand Assessment Procedure (SHAP) was developed as a means to measure the performance of upper limb prostheses. The procedure defines 26 tasks using 8 abstract objects and 14 activities to be performed by a subject fitted with a prosthetic device. Measurements of success and speed are used to calculate overall performance of prostheses. The procedures discriminate between functional and force limitations. For example, one procedure involves handling two spheres of different masses. A device may have the necessary kinematics to form a grasp around a sphere, but may not be able to lift the heavier of the two. In addition, the tests are designed around the everyday functional requirements to complete human-relevant tasks.

JP asked whether similar test procedures could be useful for dexterous robotic arms and hands. Moreover, could someone think of a set of tasks that would be a good representation of overall industry needs? For instance, the SHAP equivalent for manufacturing tasks could include $X$ abstract objects involved in $Y$ activities of daily manufacturing, and relative measurements of teaching time and throughput as compared to manual labor could be provided. Of course, the measurements could 
also be used to compare two dexterous systems independently of manual labor. A major difference between a test for humans and a test for robots is by what means intelligence is taken into account. For example, given the task, "pick and lift the heavy sphere," a human can easily interpret this operation, but robotic systems would require programmed instructions that will vary significantly between systems. The complexity of such programs could be a measure of the built-in intelligence of the system where minimal programming effort to accomplish the task indicates a high degree of intelligence.

In conclusion, there is a gap between the demand for dexterity and the available solutions to provide more dexterous robot arms and hands. As new technology emerges, it would be helpful to have performance measures to assess performance.

\section{Multimodal Tactile Sensing and Industrial Applications - Jeremy Fishel, Syntouch}

Jeremy explained the importance of the sense of touch to humans and their ability to perform dexterous tasks. Humans are much more dexterous than robots because of their sense of touch. Robots are stronger and more precise than people, and even have better performing vision capabilities... but lack the sense of touch. Jeremy referenced several studies that showed that the lack of touch in human hands resulted in loss of dexterous abilities such as buttoning a shirt, writing with a pen, or drinking from a cup.

One human study was conducted where capabilities of touch and vision were masked to see the effects of each on dexterity. In this study, touch was masked by numbing the skin. Dexterity was measured by using the hand constrained at the wrist to compress an unsteady spring using a performance measure of how much force can be applied before the spring buckles. Results were worse when neither touch nor vision was available, and steadily got better as vision was reintroduced. He also showed the results just using touch and that there was little improvement when the capability of vision was added to touch, showing that touch is the primary human sense needed for dexterity.

Syntouch has taken the approach of including all of the other types of sensing into the cutaneous capabilities of their tactile sensing system. Having everything sensed reasonably well, Jeremy explained, is better than having just one thing measured very well. Jeremy continued to describe touch in terms of cutaneous touch and proprioceptive touch. Cutaneous touch is enabled by the receptors in the skin that respond to normal and shear forces, vibrations, and temperatures. With proprioceptive touch, the actuators in muscles sense forces produced as well as the positions and velocities of parts of the musculoskeletal system. In the artificial domain, proprioceptive touch is well modeled, to the point of being better than human capability, through the use of strain gauges to measure forces and position encoders. When addressing cutaneous touch, high resolution normal force sensing is performed in a manner similar in concept to that of the use of machine vision. People are not very good at this type of cutaneous touch.

The Syntouch BioTac design approach uses all three cutaneous touch sensory modalities. It consists of a rigid core containing an array of electrodes covered with an elastomeric skin with an underlying conductive fluid, and a fingernail to hold the skin in place (which, Jeremy explained, is important for shear force sensing). The surface of the elastomeric skin contains fingerprints that are integral to sensing vibrations. The design is robust because there are no electronics within the finger's skin, a component that wears with use and is easily replaced. The BioTac can resolve force and point of contact in three dimensions. Further, it detects micro-vibrations for slip and object-texture discrimination, and can characterize objects thermally by heat transfer properties. 
Raw data, measured as changes in resistance between pairs of electrodes when the skin and fluid deform during contact, can be used to resolve normal and shear forces, torques, points of contact, and radius of curvature. The fingernail holds the skin in place and is used to measure stretching and compression around the nail base similarly to the mechanism that measures shear forces on the human fingertip. Temperature sensing is accomplished by heating the fluid to body temperature, and then thermal gradients are used to characterize and identify materials based on their thermal properties during contact. Vibration sensing is accomplished when the elastomeric skin moves across a surface. The resulting vibrations propagate through the fluid, and are measured by a pressure transducer. These sensed vibrations can then be used to detect slip and identify texture. An example of the sense of touch using vibration is the ability to sense when a screwdriver achieves insertion into the slot of a screw.

Compliance is very important for end effectors. It helps to stabilize the grasp and provides sensitivity in contact detection. However, the use of compliant material on a tactile sensor often leads to premature failure of the underlying sensor components due to stress concentrations at the boundary layer. The elastomeric skin and fluid provide needed end-effector compliance without stress on the underlying electronic components.

Jeremy explained that touch and movement are intimately coupled, and are used to perceive the environment such as identifying objects, and to provide tactile feedback for dexterity such as grip control, contact detection, and tool usage. The BioTac sensor has been used successfully to discriminate textures. Experiments were performed where 117 textures were characterized from data collected by exploratory movements involving sliding the sensor over each texture surface. Syntouch was able to discriminate all 117 textures. The sensor was sensitive enough to detect the difference between the textures of two different types of paper with 95\% accuracy, which was determined to be better than the average human performance. This research is currently being used to develop quantitative quality control measures for better ground truth than qualitative measures that are currently being used in assessing the texture quality of products. Other research in the perception area uses the sensor to give surgeons the ability to feel during laparoscopic surgical procedures.

Furthermore, Syntouch is performing research on tactile sensing for dexterity. This work uses a Kinova arm and hand, an assistive robotic platform for handicapped wheelchair patients where the patient controls a joystick to acquire and manipulate objects. The sensor system is being used to provide finger and hand position control to establish touch with multiple fingers control to within fractions of a milligram force before application of grasp force. Work in the area of fragile grasping for prosthetic hands is also being performed. Commercially-available prosthetic hands typically operate with the motors stalling on objects with high forces of up to 100 newtons, limiting the ability to grasp fragile objects. Using contact detection to modulate the commanded signals to the prosthetic hands can accommodate fragile objects, and has been demonstrated using prosthetic hands to pick up Styrofoam packaging materials without crushing them. Jeremy noted that it is much more difficult to pick up a Styrofoam peanut than an egg, because eggs are designed by nature to be structurally protective of embryos. Additional research is investigating the sensor's use in quality control, teleoperation, dexterity control, cost-reduction in making robots precise, and low-cost robots using tactile sensing.

\section{Technical Session 2 Discussions}

The current trends in making more complex hands with sophisticated sensing capability make it difficult to handle programming complexities. Ideally, it should be up to the vendors and integrators to cover the complexity of a system, and make the interface simple for the users. Alternatively, 
gripping solutions could be purely reflexive where the user programs the general grasping task, but low-level control to accomplish the task is automatic. The high- and low-level control should be decentralized to reduce the complexity for the user, but cannot be completely decoupled because there needs to be a high-level planning system, which determines the type of grasp, that must maintain communication with the low level control, which is carrying out the grasp. The necessary level of complexity from the user's perspective may vary depending on the structure of the environment. Unstructured environments, such as random bin picking, may mandate more complexity.

Alternatively, highly structured environments may require little or no complexity given that the solution is inherent in the environment. In general, the panel felt that the cost of fixturing is high, especially in the case of small production runs, and that new developments in robotic hand technology and associated perception methods will lead to its elimination in the future.

A question was posed as to whether or not performance metrics and associated test artifacts would be useful to such a young field as robotics. The panel was in agreement that there are currently no standards in place either for academia or for manufacturing in the area of robotic hand performance and grasping, but that even a rudimentary set would be useful to help assess the performance of emerging systems and associated applications. They explained that end-of-arm tooling is currently evaluated based on force, size, price, and cycle time, while there are no measures for dexterity. One panelist brought up the fact that while there have been more than 30 outcome measures proposed to evaluate prosthetic hand grasping, there is no universal standard for evaluating the performance of these hands at the clinical level either. Standards could be developed for manipulation that include test kits similar to the SHAP test kit described earlier, and that such kits could define test methods for grasping, manipulation, and even assembly operations.

When the question was posed as to what hardware or software interface standards currently exist for robot end-effectors for attachment to robotic arms, it was apparent that the only standards in the general area of end-of-arm tooling are those for mechanical connections. There are no standards for electronic connectivity, and these would be difficult to develop because of the large differences in current robotic hand designs and the number of signals needed to control them. The panel also discussed the future need to better couple the control of robotic arms to robotic hands.

An audience member asked whether there is a need for challenges, similar to DARPA's challenges, for dexterous hands. The panel agreed there was, but the DARPA challenges are focused to solve particular problem such as the Fukushima accident (which is recognized as being an important impetus for the recent DARPA disaster-response robotics challenge in terms of defining the tasks and test metrics). In the case of manufacturing, however, manufacturers and users would need to define a manufacturing problem set that would embody industry needs most likely at the low volume and high mix range of part handling. In addition to part handling, eventually robotic hands will use tools to perform operations on parts. These situations will also need to be addressed in problem sets.

Another questioner asked whether it will ever be economically practical for "universal hands" to be usable across a wide range of applications. Specifically, what is the trade-off between dexterity, complexity, and cost? It is unlikely that the cost of the mechanics will go down anytime soon, but the cost of electronics will, allowing for the devices to be produced with better, more robust sensing capabilities. Early adoption of robotic hand technology will most likely involve application where it is very expensive to use humans such as clean room environments and space. Eventually, the technology will evolve, costs will fall, and manufacturing industries will begin adopting the technology in greater numbers. In the meantime, humans are still cheaper than implementing dexterous robots in manufacturing environments. 


\section{Technical Session 3: Dexterous Arms}

\section{A. Force Guided Assembly with the KUKA Lightweight Robot - Michael Gerstenberger, KUKA Robotics}

Michael began by explaining the process of assembly, and the need for force feedback and control. The assembly process is typically broken down into four common motion tasks: part location, pegin-hole or shape insertions (which, in some cases, may be done passively through remote centers of compliance), gear meshing, and snap fitting. Due to the proximity of the robot and parts to the main assembly body, the insertion or assembly points are rarely visible. As a result, vision is of little use, and force-based search strategies must be used to help guide the robot's motions.

A force-guided search strategy consists of various motion primitives that are intended to achieve one or more goals. Common operations within a search aim to find assembly features (e.g., the surface, or holes in the surface), normalize the torque exerted on the Tool Center Point (TCP), aligning the tool with an assembly point by normalizing the TCP torque in a specific direction, maintain constant contact with the assembly surface as the tool moves, and monitor and react to force-based events (e.g., a sudden force profile change as a part breaks or snaps into place). Many of these actions may also be applied to other force-based actions such as surface finishing (e.g., grinding, polishing, cleaning, or painting) or quality assurance inspection.

Controlling the applied forces involves a two-step process. Step 1 is sensing of the incurred forces. This sensing may be done through external force/torque transducers attached to the tool flange, through joint torque sensors, or-less common these days-through monitoring motor current and inferring joint torque from the values. Step 2 involves the actual force control through a closed feedback loop of input sensor readings and output joint torques. Typically this is done through methods such as admittance control or impedance control. Admittance control can be achieved by virtually any standard position-controlled robot outfitted with a force/torque sensor, while impedance control requires the ability to command joint torques, either directly in joint space or transformed from Cartesian space. Regardless of the control mode selected, faster reactions are obviously preferable, and can be accomplished by increasing the control update rate, changing control modes or parameters (i.e., control optimization), or by reducing the inertia of the robot.

Using force control effectively eases application restrictions on part uncertainty, and allows users to be a little "sloppier" (i.e., less accurate) in terms of part placement and fixturing. When asked whether it was necessary to have a priori knowledge of the shape of the parts in order to enable component assembly, Michael responded that, although the specific shape of the part is not needed for assembly, the classification of that shape is. This classification, he explained, ultimately defines the search strategy to be used in the assembly. For instance, inserting a round peg into a round hole would use a different strategy than a similar insertion involving a square peg and a square hole.

\section{B. Part Manipulation using Sensing and Force Control - Alex Goodwin, Rethink Robotics}

Alex described Rethink Robotics flagship product, Baxter, as a robot falling into a new category of robotics defined by industry requirements for dexterity. As such, the Baxter platform offers direct manipulation as well as integral vision and force sensing of two highly dexterous 7 DOF arms. This new technology is designed to work alongside people performing human-scale tasks at human cadence, eliminating the need for safety cages. This is accomplished through a series of elastic actuators incorporated into all 14 arm joints. Alex explained that Rethink Robotics' Baxter uses force 
sensing both to detect collisions for human safety and for teach-by-example programming. Force control is achieved using a series of elastic actuators, which allows for the robot to measure force and control the applied force/stiffness quickly.

The design of Baxter was driven by human-robot interaction and task performance requirements. Alex compared the capabilities of humans when performing such tasks as capping a bottle, inserting a pin, or performing a finishing operation, with the current capabilities of very repeatable industrial robots that depend on end effector technologies such as remote center of compliance and specialized tools for accomplishing the same tasks. Baxter is intended to be used to approach such tasks using force-controlled strategies that are augmented with vision capabilities. Dexterity, Alex explained, is important for assembly, finishing, and machine loading. Assembly requires precision placement of parts in position control. Force control allows for slop by compensating through sensor feedback. Finishing operations require precision placement and control, and a highly structured environment.

In summary, Alex described the human robot interaction capabilities designed into Baxter. These include human presence detection, a user interface on the robot arms with a display on the robot face, and the ability to train objects and tasks by direct movement of the arms. Baxter is capable of behavior-based intelligence, force sensing and force control at each joint, vision-guided movement, and visual object identification towards performing dexterous manipulation tasks with a payload of up to $2.3 \mathrm{~kg}$ per arm using interchangeable end effectors. The maximum $1 \mathrm{~m} / \mathrm{sec}$ arm speed yields 6-10 pick and place operations per minute.

\section{Bilateral Manipulation Applications in Industry - Tim DeRosett, Yaskawa Motoman Robotics}

Motoman, Tim stated, was pleased to see that this workshop was focused on grippers as well as on dexterous robotic arms, because the dexterity of a robot is only as useful as the end effectors will allow. Whether integrated with tactile sensors like the Schunk robotic hands, mechanically underactuated like the Robotiq, or one of the newly-developed robotic hands coming out of the DARPA robotics challenge, robots succeed or fail by the gripper, and continued work on grasping is paramount. The resolution and precision of a robot is ultimately defined by the ability to achieve fine motions with minimal jitter. Dexterity is often perceived as economy of motion, or "nimbleness." Economy with respect to motion is difficult to define, but one can say it is smooth and fluid motions with energy/effort efficiency. "You know it when you see it," Tim explained. For example, if one were to watch a person sorting beans or electronic components, they would see the human exhibit economy of motion. The motions are very deft with the appearance that the hands are hardly moving at all. In contrast, delta robots have incredible precision in the work volume, but they do not exhibit "economy" because they have several arms swinging around for a simple transfer path. Economy of motion relates the desired end effector motion to the robot motion required to achieve it. Cup stacking is an example of manipulation and grasping in which speed is achieved through economy of motion. Some people can stack cups very quickly and very precisely using a sequence of coordinated and efficient motions generated by their two human powered manipulators. Robots are currently incapable of competing with human speeds to perform such tasks.

The coordination of multiple arms allows for greater dexterous manipulation capabilities. Multiple arms can be controlled separately or jointly, depending on the task requirements. Arms with 7 DOF provide a huge advantage over traditional arm designs having fewer degrees of freedom, and

represent a breakthrough in agility. Similarly, dexterous grippers provide more flexible capabilities 
and more cost effective options for flexible tooling. Safe, collaborative robots suffer from a tradeoff with throughput because of speed limitations when working close to humans.

Motoman, Tim explained, defines the elements of dexterity as coordination and agility. In terms of robotics, coordination applies only to bilateral manipulators. To apply metrics to coordination one needs to determine a level of hand-hand coordination. Any test of dexterity should include cooperative tasks where both arms work together. Additional metrics will need to be considered that decouple the manipulator coordination from the grippers. The agility for a given robot is the ability to reach the greatest possible volume with the greatest number of orientations and configurations. While agility is related to the number of DOF, a robot can have kinematic redundancy but still be limited in its accessible volume. With regard to agility, Tim also defined footprints as a ratio of the root physical volume to the working volume.

\section{Technical Session 3 Discussions}

In the previous technical session concerning robotic hands, the audience was told that it was currently not possible to classify the metrics of dexterity. Turning the focus now toward robot arms, an audience member asked how would (or should) one attempt to measure dexterity of arms. Referring back to Tim DeRosett's presentation, one panelist suggested that the volume of reachability would likely be a good starting point (e.g., as described in Eugene Rivin's Mechanical Design of Robots ${ }^{3}$ ). This volume, however, can get very complex very quickly if the person doing the evaluation also starts factoring into the equation variables such as DOF and null spaces. Furthermore, another panelist offered, the level of dexterity may also be evaluated according to the number and types of applications that a particular robot is capable of supporting or performing. However, this also stresses the importance of the capabilities of the tooling attached to or complementing the robot. An alternative concept for consideration is that of "usable dexterity," in which the integration of additional axes in order to achieve higher physical dexterous performance also adds to the complexity of controlling the robot. For such classifications, attention would have to be focused on energy and effort optimization. This is, however, an ongoing issue with robots in general, and is an integral component of usability and performance evaluation and optimization. Taking force-guided operations as an illustrative example, optimizing the speed of the closed force-feedback control loop (i.e., evaluate, test, and move) and overall performance often requires low-level tricks or hacks such as triggers operating below the program interpreter that are either hidden or simply not available to the user.

Another question raised awareness of the viability of adopting truly dexterous robots within the manufacturing industry. The panel members shared three different aspects of approaching this problem. The first suggested that robot vendors and integrators should start at a simple, easy-to-attain state of dexterity, and then take a stepped and metered approach to introducing options to new markets and applications. The second approach was to find and feature a "killer application" that highlights dexterous robotics as a serious automation solution. It was posited that one such application could be gearbox assembly, which is often touted as being a very difficult task for human and robot alike. However, it was pointed out that a fundamental problem with a killer application is that it pushes aside awareness of the difficulties of programming. Software will always be complex, especially as the applications and capabilities of the software grow. Vendors will, again, likely have to focus on who will be using their technology solutions, and who will be responsible for the integration of these technologies into the manufacturing environment. Finally, a third perspective suggested that the bulk of the responsibility for common dexterous robot tasks should be shifted away

3 Rivin, E.I. Mechanical Design of Robots. Mcgraw-Hill. 1987. ISBN-13: 978-0070529922. 
from end-user programmed tasks toward canned solutions embedded within the dexterous robot system. While more complex tasks would necessarily require more user input, it is not unreasonable to expect dexterous robot manufacturers to provide solutions for simple problems.

Dexterity in robotics has been-and continues to be-a hot topic of active research. This is made abundantly clear by the sheer volume of effort being applied to anthropomorphic robot hand research. However, these solutions are, more often than not, one-off answers with little chance of commercial success, and do not have the robustness or user base necessary to ensure longevity outside of a laboratory. A fundamental question is thus: how is industry to actually drive the transition of research solutions into real world solutions? One possible answer is to involve crowd-sourcing efforts. Open-source communities may help bridge the gap between research effort and commercial viability by building user bases and technological improvements. However, even the open source community is built on standards — such as established programming languages — that provide a framework for development. As mentioned previously, such standards do not yet exist for dexterous robotics. There are also additional costs associated with joining such communities that act as a barrier to entry. For example, Rethink Robotics’ Baxter at $\$ 22,000^{4}$ is inexpensive when compared with the asking price of comparable robot arms. However, this price is far from affordable to a hobbyist. At the end of the day, vendors must still follow a business model. A fundamental required change is to adjust these business models to incorporate the benefits of being associated with the research community.

\section{Summary}

A NIST-sponsored workshop identified the challenges manufacturers are facing in production environments characterized by low volumes of parts with high mixtures of part types, a situation that is typical of small and medium sized manufactures but is also prevalent among larger manufacturers that produce many product types in the form of small batch production runs. The workshop also discussed the automation requirements to support these manufacturing challenges and the associated emerging technologies through the participation of key industry leaders in dexterous grasping, robot arm technology, and flexible manufacturing and an open format discussion with all workshop participants.

Automation requirements focused on highly dexterous robotic systems that could acquire a multitude of objects in any number of orientations with the speed and actuation necessary to grasp adeptly and decisively. These automation systems must incorporate force and tactile sensing enabling a flexibility that is "blind to shape" in order to use the sense of touch to acquire as humans do. Other requirements included the need for portable automation to support large footprint manufacturing operations as well as the capability to dispatch a robot from process to process throughout a plant.

A view into the current and leading edge adaptive grasp technologies in gripping and robotic hands revealed a developing transition from conventional customized gripping solutions to intelligent gripping technologies for examination, flexibility, and adaptation to varying part geometries with the growing emergence of robotic hand technologies from research and development environments. Advances in robot arm technology are also being made to support the dexterity needed for flexible manufacturing by integrating force sensing technology directly into robot joints to ease application restrictions on part uncertainty and to compensate for inaccuracies in terms of part placement and

4 The retail price of Rethink Robotics' Baxter as of 1/24/2013 is listed at $\$ 22,000$. This price is for the robot platform only. Equipping Baxter with the grippers and a pedestal base demonstrated at Automate increases the cost to $\$ 27,000$. 
fixturing and assembly operations. In addition, these new robot arm technologies are being designed with force control and compliance characteristics that enable them to work alongside people, performing human-scale tasks at human cadence, eliminating the need for safety cages.

Discussions emerged around the need for performance metrics and test methods for this new generation of robotic hands and arms, as well as for integrated systems consisting of both. A framework to evaluate tasks and parts was identified as a means to characterize the effectiveness of solutions and the use of challenges was discussed as a viable mechanism to drive research and development. A rudimentary set of performance metrics and associated test artifacts was identified as a useful tool in helping to progress such a young sub-field of robotics. Workshop participants agreed that dexterous robotics will continue to be a topic of active research due to the sheer volume of work coming from universities with the unanswered question being: how will industry drive the transition of research solutions into real world solutions?

The results of this workshop will be used as input for a roadmap being drafted by NIST aimed at progressing standards and measurement science in the area of dexterous manipulation. This report as well as the forthcoming roadmap will be made available on the Dexterous Manipulation for Part Grasping and Assembly project website (http://www.nist.gov/el/isd/ps/dexmanpartgraspassem.cfm). 


\section{Appendix}

\section{A. Workshop Announcement}

\section{Dexterous Manipulation for Manufacturing Applications Workshop}

The ability of humans to manipulate a wide range of objects with great dexterity and precision enables them to operate productively in the world. People can build things, take them apart, and determine many part properties simply by touch. This is currently beyond the capabilities of even the most sophisticated robots, and is a major obstacle to moving robots into small and medium-size enterprises. This obstacle also places limitations on large manufacturers interested in implementing robotics for small-batch production. Smaller production runs cannot justify the cost associated with conventional practices such as fixed tooling and purpose-built manipulation systems.

The National Institute of Standards and Technology has organized a workshop in conjunction with the 2013 Automate and ProMat trade shows in Chicago, IL. The goal of the workshop is to address the application of dexterous robot technologies to meet the application needs of small- and medium-sized manufacturers, and to promote the greater theme of applying dexterous robotics toward flexible manufacturing. Join us on January 24th from 10:30 AM until 5:00 PM for three technical sessions featuring presentations from industry leaders in dexterous grasping, robot arm technology, and flexible manufacturing. Each session concludes with an open-format panel discussion where all are welcome to discuss the needs and opportunities for dexterous manipulation in next-generation manufacturing systems.

The workshop results will be published in a report that captures key discussions, including

- desired application scenarios requiring more dexterous manipulation

- current state-of-the-art versus desired technological capabilities

- high priority advances needed to enable industry's needs for more dexterous robots

- performance metrics to support the advancement of dexterous robots

These results will provide valuable input to a roadmap being drafted by NIST aimed at progressing standards and measurement science in the area of dexterous manipulation. 
B. Workshop Agenda

10:30 - 10:35 Jeff Burnstein (RIA) - Welcome

10:35 - 10:40 Joe Falco (NIST) - Introduction

\section{Technical Session 1: Industry Perspective on Dexterous Manipulation}

$10: 40-11: 00$

\section{Mark Lewandowski (Procter \& Gamble)}

Title: Robotic Gripping and Manipulation Needs at P\&G

Abstract: As the world's largest consumer goods company, Procter \& Gamble has many needs in its manufacturing processes for the application of dexterous robot technologies. This presentation will highlight some example applications and discuss $P \& G$ 's needs for robotic grippers and manipulators that are human-like, reliable and affordable.

Bio: Mark Lewandowski is a Controls and Information Systems Technology Leader in Corporate Engineering for Procter \& Gamble. He has 22 years of machine controls experience with Procter \& Gamble in high speed converting, packaging and robotics applications. Mark is the leader of the P\&G Robotics Technical Network that is chartered to develop standard approaches for robotics technology throughout $P \& G$, and is also responsible for identifying new and innovative robotic technologies to leverage within the company. Mark is also a member of the RIA Board of Directors and participates in several industry standard development groups. He has a BS in Electrical Engineering from Mercer University in Macon, GA.

\section{$11: 00-11: 20$}

\section{Craig Battles (Boeing)}

Title: Assembly and Automation Technology

Abstract: The Assembly and Automation Technology presentation describes aerospace business drivers at Boeing and their relationship to current and next generation production methods. The talk will emphasize the aspect of those new capabilities that would most benefit from dexterous manipulation technologies.

Bio: Craig earned physics degrees in the early 1970s from the University of Washington and joined Boeing in 1974. In his nearly 40 year career at Boeing he has designed, developed and tested production systems for components of Airborne Warning and Control aircraft, Air Launch Cruise and anti-aircraft missiles and every Boeing Commercial Airplane model from the 737 on. Craig holds a number of patents in manufacturing processes, sensors and RFID systems. He is a Technical Fellow of Robotics and Automation with Boeing Research and Technology.

\section{1:20-11:40}

\section{Clay Flannigan (Southwest Research Institute)}

Title: Material Handling in Unstructured Environments: Challenges and Opportunities for Small and Medium Manufacturers

Abstract: The talk will present a method to characterize robotic material handling problem complexity as it relates to small and medium manufacturers. Current state-of-the-art technical approaches to the unstructured manipulation problem will be discussed along with some practical examples.

Bio: Clay Flannigan is the Manager of Robotics and Automation Engineering at Southwest Research Institute. His group focuses on applied research for robotics in manufacturing and 
industry. His research interests include sensing and perception for robot guidance including manipulation and autonomous vehicles. He holds a Master's Degree in Mechanical Engineering from Case Western Reserve University.

$11: 40-12: 00$

Discussion

$12: 00-1: 00$

Lunch Break

\section{Technical Session 2: Robotic Hands}

1:00-1:20

\section{Jesse Hayes (Schunk)}

Title: Robotic Gripping - What is the Future

Abstract: The talk will give an overview of robotic hands at SCHUNK. First raising the question of what could the future of robotic gripping look like, followed by a general look at what is the perfect gripper (human hand?) and some of its limitations. We will then look at the market trends in robotic gripping from Gripping to Smart Gripping and then to the future of Intelligent Gripping (this represents the past present and future of gripping). Finally, we will take a brief technical look at the state of the art with the SCHUNK robotic hand product line.

Bio: Jesse Hayes is the Automation Group Manager for SCHUNK, the worldwide competence leader in clamping and gripping technology. Jesse has a strong technical background and a passion for technology. With over 15 years of engineering experience, Jesse brings exceptional knowledge to the SCHUNK team. He has been a part of the SCHUNK Automation team for over 11 years and played a critical role in developing SCHUNK into a global leader in the automation world.

$1: 20-1: 40$

Jean-Philippe "JP"Jobin (Robotiq)

Title: Useful Robot Dexterity for the Factory

Abstract: In this presentation, JP Jobin will share with the audience insights gained in his past five years of installing industrial robotic hands into factories. Starting from end-user needs, he will present different approaches to hand design. Future R\&D trends will be exposed to open up on a discussion around the benchmarking of dexterous manipulation systems.

Bio: JP Jobin is the Vice President of research and development at Robotiq, a company that makes industrial robotic hands used in factories needing a high level of flexibility. JP holds an MSc in mechanical engineering completed at the Laval University Robotics Laboratory, in Canada.

1:40 - 2:00

\section{Jeremy Fishel (SynTouch)}

Title: Multimodal Tactile Sensing and Industrial Applications

Abstract: Touch and movement are intimately coupled. As evidenced in clinical studies, cutaneous receptors are not only required for tactile perception of objects, they are essential for the dexterity of the human hand; both human and robot hands without tactile sensing will

\footnotetext{
${ }^{5}$ Jean-Philippe Jobin filled in for Samuel Bouchard, who was unable to attend.
} 
be similarly disabled. While many decades of research have gone into the development of tactile sensors, few designs have possessed the requisite sensitivity and robustness to be suitable for commercial applications. Here we introduce the BioTac ${ }^{\circledR}$ (SynTouch, LLC), a uniquely robust, compliant, finger-like multimodal sensor modeled after the cutaneous tactile sensory capabilities of the human fingertip (force, vibration and temperature). Industrial applications of this technology currently underway at SynTouch, LLC include prosthetics, telerobotics, and tactile object perception.

Bio: Jeremy Fishel received a B.S. in Mechanical Engineering ('05) from California State University Long Beach, before completing two M.S. degrees (Biomedical Engineering in '07; Aerospace and Mechanical Engineering in '09) at the University of Southern California. He received a PhD from the Biomedical Engineering department at USC ('12) for his work on fluidbased tactile vibration sensing and the development of Bayesian exploration. He is also a cofounder of SynTouch, which manufactures biomimetic tactile sensors, and has been the lead systems engineer on the development of the BioTac technology since the company's founding in '08. As Director of Research at SynTouch, Dr. Fishel's interests are in developing applications of tactile sensing in robotics, telerobotics and prosthetics.

2:00-2:20

Discussion

2:20 - 2:40

Break

\section{Technical Session 3: Dexterous Arms}

2:40-3:00

\section{Michael Gerstenberger (KUKA Robotics)}

Title: Force Guided Assembly with the KUKA Lightweight Robot

Abstract: Force-guided assembly is a robotic application that is being enabled by the newest highly sensitive robots. The requirements of assembly operations are presented and mapped to features needed to perform robotic assembly. The features of the KUKA Lightweight Robot (LWR) are discussed in the context of these assembly operation requirements. Specific assembly operations are demonstrated with the LWR.

Bio: Michael Gerstenberger is a senior engineer with KUKA Robotics, responsible for introducing advanced KUKA technologies into the North American market. His focus areas include the KUKA Lightweight Robot (LWR), Force/Torque Control, Robot Sensor Interface, and safety technologies. He has over 27 years of experience in robotics, specializing in robot kinematics and dynamics, trajectory generation, and servo control. He has a BSE from the University of Michigan and SM and EE degrees from the Massachusetts Institute of Technology.

$3: 00-3: 20$

\section{Alex Goodwin (Rethink Robotics)}

Title: Part manipulation using sensing and force control

Abstract: Rethink Robotics's flagship product, Baxter, uses environmental sensing and force control to enable part manipulation similarly to people's methods. People use their sense of force to increase their manufacturing effectiveness and vision to gain more knowledge about their environment. This presentation will cover example tasks workers regularly perform 
and how force control can be used to increase a robot's capabilities to work in unstructured environments.

Bio: Alex Goodwin is Director of Product Management at Rethink Robotics. Alex's career has focused on bringing breakthrough technologies to market within startup environments. He successfully led product management and development at Brontes Technologies, a 3M Company, and SensAble Technologies. Alex holds an MBA from Harvard and a Mechanical Engineering degree from MIT.

\section{$3: 20-3: 40$}

\section{Erik Nieves ${ }^{6}$ (Yaskawa Motoman Robotics)}

Title: Bilateral manipulation applications in industry

Abstract: The introduction of bilateral manipulation has opened many new applications for robotic automation. These tasks were formerly outside the realm of automation due to various constraints. This talk will review the use of dual arm robots for new tasks and discuss the technical obstacles yet to be overcome for wider adoption. Recommendations for areas of research and new metrics for dexterity will be proposed.

Bio: Erik Nieves is currently Technology Director for Yaskawa Motoman Robotics, responsible for the corporate strategic technology roadmap with special focus on emerging applications. During his 20+ year tenure at Motoman, Erik has held a variety of leadership positions in the U.S. and abroad. A passionate advocate for robotics, Erik is an active member of the Robotics Industries Association (RIA), where he serves on the Education subcommittee and on the "Ask the Experts" forum. Erik has published many articles on advances in robot technology, including dexterous manipulation, multiple arm control, controls architecture, and robotic metrology. Erik holds a B.S. degree in Mathematical Physics from Southwestern Adventist University (1990).

\section{3:40 - 5:00 \\ Discussion/Wrap-up}

\footnotetext{
${ }^{6}$ Tim DeRossett filled in for Erik Nieves, who was unable to attend.
} 